\title{
Synthesis and properties of monodisperse conductive core-shell latexes
}

\author{
Lee Y. Wang ${ }^{a^{*}}$, Yi-Jun Lin ${ }^{\mathrm{b}}$, and Wen-Yen Chiu ${ }^{\mathrm{b}}$ \\ ${ }^{a}$ Center for Condensed Matter Sciences, National Taiwan University, Taipei, Taiwan \\ ${ }^{b}$ Department of Chemical Engineering, National Taiwan University, Taipei, Taiwan
}

\begin{abstract}
Monodisperse polystyrene/polyaniline core-shell latexes have been synthesized using cationic spheres as polymer core. These conductive colloids were characterized by IR, SEM and TEM. The conductivity percolation threshold was found to be around 3 wt $\%$ of polyaniline.
\end{abstract}

Keywords: Polyaniline; Conductive colloids; Core-shell; Composite; Polystyrene

\section{Introduction}

Polyaniline (PANI) is generally categorized as a rigid and infusible material and only slightly soluble in a limited number of solvents, such as dimethylformamide [1], $\mathrm{N}$ methylpyrrolidone [2] and concentrated sulfuric acid [3]. An approach to overcoming these disadvantages is the preparation of conducting polymers in colloidal form. Synthesis of stable conductive polymer latex is usually performed by chemical oxidative polymerization of the appropriate monomers in the presence of a water-soluble stabilizer, which physically adsorbs or chemically grafts on latex surfaces providing efficient steric repulsion between particles. An alternate route for preparing colloidal conducting polymers involves coating latex particles with a thin layer of conjugate polymer to form conductive composites with a core-shell morphology [4-6]. The polymer core is usually a neutral or anionic non-conducting particle. In this article we describe the synthesis of stable core-shell polyaniline colloids by the use of cationic polystyrene latex as a core particle.

\section{Experimental}

In a typical experiment, $45 \mathrm{ml}$ of polystyrene (PS) latex was diluted with $135 \mathrm{ml}$ of deionized water, acidified with $\mathrm{HCl}$ to $\mathrm{pH} 0.7$, mixed with $0.68 \mathrm{~g}$ of sodium dodecyl sulfate (SDS), and stirred for $12 \mathrm{~h}$ to allow the attainment of the adsorption-desorption equilibration. Purified aniline was added via syringe and this dispersion was stirred for 1 $\mathrm{h}$, followed by the addition of aqueous ammonium persulfate solution to start the reaction. Polymerization was allowed to proceed at $5{ }^{\circ} \mathrm{C}$ for $6 \mathrm{~h}$ and then at room temperature for another $18 \mathrm{~h}$.
The conductivity of PS/PANI core-shell latex was measured on compressed pellets of the water-washed and vacuum-dried samples using four-probe technique at room temperature. The particle sizes and morphologies of colloids were examined by scanning electron microscopy (Jeol JSM-6300) and transmission electron microscopy (Hitachi H-7100). FTIR experiments were performed on a Nicolet 750 instrument.

\section{Results and discussion}

The synthesis of cationic polystyrene latex was carried out by surfactant-free emulsion polymerization method using azobis(isobutylamidine hydrochloride) as initiator. The SEM micrograph of the obtained PS latex showed a fairly narrow size distribution, as shown in Fig. 1. The number-average particle diameter is $0.46 \pm 0.01 \mu \mathrm{m}$. The solid content was determined gravimetrically to be 3.94 wt $\%$.

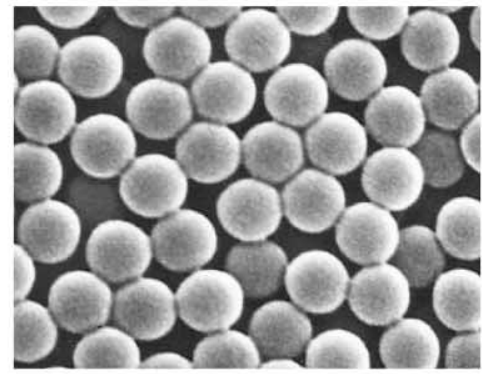

Fig. 1. SEM micrograph of cationic polystyrene latex.

The PS/PANI composite latexes were prepared by the polymerization of aniline in aqueous hydrochloric acid using $\left(\mathrm{NH}_{4}\right)_{2} \mathrm{~S}_{2} \mathrm{O}_{8}$ oxidant in the presence of PS spheres.

\footnotetext{
* Corresponding author. Tel: +886-2-2369-6221; fax: +886-2-2365-5404; E-mail: leewang@ccms.ntu.edu.tw
} 
A wide range of stabilizers, e.g. poly (ethylene oxide) [7], poly (vinyl alcohol) [8], poly(vinyl alcohol coacetate) [9], poly(2-vinylpyridine-co- $p$-aminostyrene) [10], poly(sodium styrene sulfonate) [11] and colloidal silica particles [12] have been reported by several research groups for making stable polyaniline colloids. In order to afford better stability, the monodisperse cationic PS particles were equilibrated with SDS anionic surfactant overnight prior to the polyaniline coating. A typical SEM picture of the obtained composite colloids is illustrated in Fig. 2(a). All particles are in spherical shape and have nearly monodisperse size distribution. The number-average particle diameter is almost the same as the size of PS core. These observations exclude the possibility of the formation of pure polyaniline particles during the polymerization process of aniline. Fig. 2(b) shows a TEM micrograph of PS/PANI latexes. It indicates a thin PANI ring around PS sphere, providing the direct evidence of the core-shell morphology. (a)

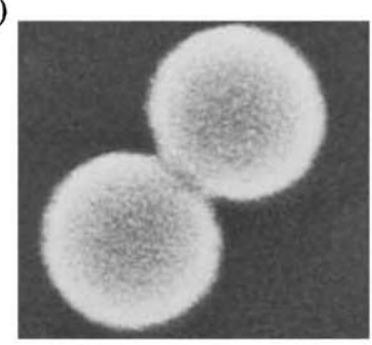

(b)

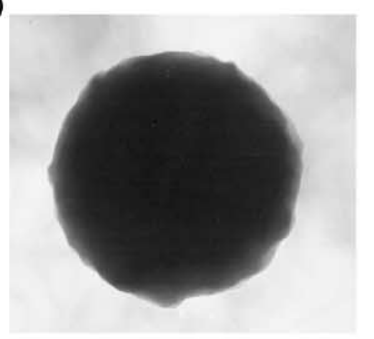

Fig. 2. (a) SEM and (b) TEM micrographs of PS/PANI composite latex.

The FTIR spectra of polystyrene, polyaniline in emeraldine salt form, and PS/PANI composite particle are presented in Fig. 3. Obviously, the spectrum of latex particle displayed a combined feature of infrared absorption of PS and PANI, indicating the coexistence of both polymers. The penetration depth of IR beam ranging from 1 to $10 \mu \mathrm{m}$ is much larger the particle diameter. However, Fig. 3(c) noticeably exhibited a strong absorption band at $1110 \mathrm{~cm}^{-1}$ attributed to PANI, even though the PANI loading on this latex is only $3.0 \mathrm{wt} \%$. The result indirectly reconfirmed the core-shell morphology of the assynthesized latex composite.

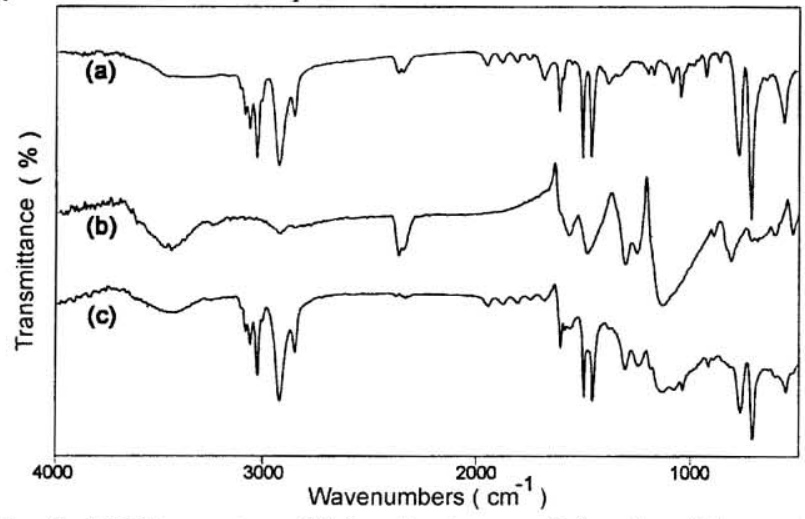

Fig. 3: FTIR spectra of (a) polystyrene; (b) polyaniline and (c) PS/PANI composite latex containing $3 \mathrm{wt} \%$ of PANI.
The electrical conductivity of the composite versus the weight fraction of polyaniline is plotted in Fig. 4. The conductivity increases rapidly with increasing PANI loading and it is relatively high even when the PANI content is low. The electrical conductivity percolation threshold is in the range of $1-3 \mathrm{wt} \%$ of PANI. This value is slightly lower than that of PS/PVP/PANI composites prepared previously by Armes et al. [6]. As the mass loading of PANI reaches $\sim 5 \mathrm{wt} \%$ the conductivity of this material is comparable to that of pure polyaniline powder.

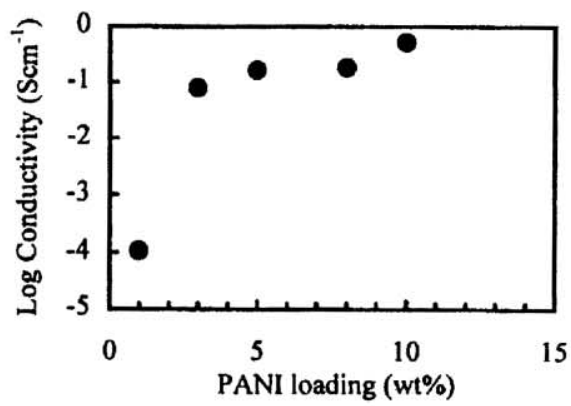

Fig. 4: Electrical conductivity of PS/PANI latexes as a function of weight fraction of PANI.

In conclusion, this work has shown that stable submicronic conductive core-shell latexes with a wide range of PANI loading can be easily prepared by the use of cationic polystyrene latex with the adsorption of SDS surfactant as core particle. These composites possess comparable electrical conductivity as pure polyaniline and a low percolation threshold of around $3 \mathrm{wt} \%$.

\section{Acknowledgement}

This work was supported by the National Sciences Council of Republic of China under Grant number of NSC 88-2113-M-002-007.

\section{References}

[1] M. Angelopoulos, A. Ray and A. G. MacDiarmid, Synth. Met. 21 (1987) 21.

[2] M. Angelopoulos, G. E. Asturias, S. P. Ermer, A. Ray, E. M. Scherr, A. G. MacDiarmid, M. Akhtar, Z. Kiss and A. J. Epstein, Mol. Cryst. Liq. Cryst. 160 (1988) 151

[3] A. Andreatta, Y. Cao, J. C. Chiang, P. Smith and A. J. Heeger, Synth. Met. 26 (1988) 383.

[4] M. A. Khan and S. P. Armes, Adv. Mater. 12 (2000) 671.

[5] F. M. Huijs, F. F. Vercauteren, B. de Ruiter, D. Kalicharan and G. Hadziioannou, Synth. Met. 102 (1999) 1151

[6] C. Barthet, S. P. Armes, S. F. Lascelles, S. Y. Luk and H. M. E. Stanley, Langmuir 14 (1998) 2032.

[7] S. P. Arms and M. Aldissi, J. Chem. Soc. Chem. Commun. (1989) 88.

[8] S. P. Armes, M. Aldissi, S. F. Agnew and S. Gottesfeld, Mol. Cryst. Liq. Cryst. 190 (1990) 63.

[9] S. Maeda, D. B. Cairns and S. P. Armes, Eur. Polym. J. 33 (1997) 245.

[10] N. Gospodinova, P. Mokreva and L. Terlemezyan, J. Chem. Soc. Chem. Commun. (1992) 923

[11] B. Vincent and J. Waterson, J. Chem. Soc. Chem. Commun. (1990) 683.

[12] J. Stejskal, P. Kratochvil, S. P. Armes, S. F. Lascelles, A. Riede, M. Helmstedt, J. Prokes and I. Krivka, Macromolecules 29 (1996) 6814. 